\title{
Efficacy of Pulse Co-oximeter in Hemoglobin Estimation: A Non Invasive Method
}

\author{
Jayalaxmi Yadav $\mathrm{K}^{1 *}$, Bharat $\mathrm{C}^{1}$, Shridevi $\mathrm{SH}^{1}$ and Udayshankar ${ }^{2}$ \\ ${ }^{1}$ Department of pathology, Vijayanagara Institute Of Medical Sciences, Ballari, Karnataka, India \\ ${ }^{2}$ Blood bank, District Hospital, Vijayanagar Institute Of Medical Sciences Ballari, Karnataka, India
}

\begin{abstract}
Background: Total Hemoglobin $(\mathrm{Hb})$ measurement is one of the most common and important parameter that is investigated in the laboratory. This non invasive method allows pain free continuous online patient monitoring with minimum risk of infection and facilitates real time data monitoring allowing immediate clinical reaction to the measured data. The objective of the study is to test the efficacy of pulse co-oximeter .

Methods: Present study is a prospective comparative study. A total of 261 patients enrolled for the study after their consent. Non invasively measured $\mathrm{SpHb}$ values and invasively measured $\mathrm{Hb}$ values were then compared by using Bland Altman statistical analysis .

Result: Pulse co-oximeter recorded/displayed $\mathrm{SpHb}$ in $90.8 \%$ patients and did not display $\mathrm{SpHb}$ in $9.2 \%$ of patients. Out of which $73-75 \%$ patients $\mathrm{Hb}$ showed variation of less than or equal to $2 \mathrm{gms}$ and $20-24 \%$ patients Hb showed variation of more than 2 gms. On Bland Altmann comparative analysis of $\mathrm{SpHb}$ and $\mathrm{Hb}$ revealed bias and limits of agreement was $0.2+3.3 \mathrm{gm} / \mathrm{d}$ and $95 \%$ of the measurements fell within two standard deviation of the mean difference and $\mathrm{P}$ value $<0.001$ represents good correlation between SpHb and $\mathrm{Hb}$ of Lab1 and Lab 2 .

Conclusion: Pulse co-oximeter can be used as a screening tool for $\mathrm{Hb}$ measurement . It determines Hb instantly and non invasively with $73-75 \%$ of the values show variation of less than or equal to 2 gms. Further studies are needed to determine financial aspects and needs upgradation in terms of accuracy of the instrument.
\end{abstract}

Keywords: Pulse Co-oximeter, Non Invasive Method, Bland Altmann Analysis

\section{Introduction}

Hemoglobin $(\mathrm{Hb})$ can be measured on a variety of devices using different principles of operation. Non invasive pulse co-oximeter represents the latest development in hemoglobin measuring technology. The technology uses principles similar to pulse oximetry in measuring total hemoglobin, oxyhemoglobin, carboxyhemoglobin and methhemoglobin. The pulse oximeter works by illuminating light into the tissues and sensing the amount of light absorbed. The same methodology is used by laboratory hemoglobinometers to measure hemoglobin concentration. Because both devices work in the same way, efforts were made to modify the pulse oximeter to also measure hemoglobin concentration. Currently there are two commercial pulse co-oximeters (Masimo Rainbow SET and OrSense NBM-200MP) that measure total hemoglobin concentration and one (Masimo) that also measures methemoglobin and carboxyhemoglobin ${ }^{[1]}$. The technology is noninvasive and provides continuous monitoring in comparison to invasive and discrete techniques used in other methods, pulse co-oximeter purportedly provides an advantage in patient care. In present scenario invasive methods are used to measure the $\mathrm{Hb}$ concentration, $\mathrm{Hb}$ measurement using an automated analyzer in a clinical laboratory is the gold standard method [2]. Apart from the discomfort of ejecting blood samples an added disadvantage of this method is delay between blood collection and its analyses which does not allow real time patient monitoring in critical situations. The purpose of this research is threefold: a) To study accuracy of pulse co-oximeter (Masimo Rainbow SET) by comparing its results with hematology analyzer b) To review the various underlying principles used in measuring $\mathrm{Hb}$ c)To discuss issues in implementing pulse co-oximeter into a laboratory or hospital

\section{Materials and Methods}

The present study is a prospective comparative study done over a period of two months during October and November 2016. The study was conducted in central laboratory district hospital VIMS Ballari. After approval from ethical committee VIMS, informed consent was obtained from patients prior to their enrollment. On the basis of previous study performed by Allard ${ }^{[3]}$ et al, we did hemoglobin estimation on 261 patients by pulse cooximeter(SpHb), Lab1-central lab Sysmex hematology analyzer( $\mathrm{Hb})$ and Lab 2-Medall laboratory hematology analyzer( $\mathrm{Hb})$. $\mathrm{SpHb}$ estimation by pulse co-oximeter was done while subjects were quiet and sitting upright. Sensor 
of pulse co-oximeter was covered with an opaque shield to prevent optical interference.

Immediately following the noninvasive testing, a venous blood sample was obtained by venipuncture of the median cubital vein of the non dominant arm with a disposable syringe and then transferred to $2 \mathrm{ml}$ vacuum tube containing EDTA. Venous blood samples were transported at room temperature and analyzed for reference hemoglobin value with Lab1-central laboratory Sysmex hematology analyzer and Lab 2-Medall laboratory hematology analyzer as per clinical and Laboratory Standard Institute guidelines and manufactures directions for use with in 24 $\mathrm{hr}$ of collection. The laboratory analyzer was calibrated daily as per the manufacturer's recommendations and good laboratory practice.

This newly developed pulse co-oximeter (Masimo Rad 57) is an optical sensor system uses multiple wavelengths of light for $\mathrm{Hb}$ measurement ${ }^{[2]}$. The $\mathrm{Hb}$ sensor developed for this research is fully integrated into a wearable finger clip. The devise is based on technology known as occlusion spectroscopy which uses an optical measurement platform combined with a ring shaped pneumatic probe that fits on the finger ${ }^{[3]}$ (Figure 1).

Statistical Analysis: Statistical analysis was performed to determine the relationship between $\mathrm{SpHb}$ and the standard laboratory $\mathrm{Hb}$. We calculated the correlation coefficient (r) and coefficient of determination (r2). Agreement between the laboratory $\mathrm{Hb}$ and $\mathrm{SpHb}$ was evaluated as described by Bland and Altman ${ }^{[5]}$. The accuracy of the $\mathrm{SpHb}$ compared with that of the laboratory $\mathrm{Hb}$ was calculated using the accuracy root mean square (Arms) with the formula Square root of( mean bias square + SD square) ${ }^{[6,7]}$. All statistical analysis was performed with SPSS Version 19.0, with the statistical significance set at $\mathrm{P}<0.005$.

\section{Results}

Conducted prospective study on 261 out patients visiting laboratory for routine hemoglobin estimation . Patients belonged to varied age group and males were $70.9 \%$, females were $29.1 \%$. Hb estimation was done by 3 different methods on 261 patients

1) $\mathrm{SpHb}$ by pulse co-oximeter (Masimo)

2) Lab1- central lab district hospital by sysmex hematology analyzer

3) Lab 2- Medall lab hematology analyzer

These 3 different methods were compared and statistically analyzed. Among 261 patients pulse co-oximeter did not record $\mathrm{SpHb}$ values in 24 patients. Pulse co-oximeter can record/display $\mathrm{SpHb}$ in $90.8 \%$ patients and did not display $\mathrm{SpHb}$ in $9.2 \%$ of patients due to unknown reasons. Hence a total of $n=237$ cases were statistically analyzed and 24 patients were excluded from the study. On comparison of $\mathrm{SpHb}$ values with $\mathrm{Hb}$ of lab1, variation of less than or equal to $2 \mathrm{gms}$ is seen in $75 \%$ of patients and variation of more than $2 \mathrm{gms}$ is seen in $20 \%$ patients(Table 1). On comparison of $\mathrm{SpHb}$ values with $\mathrm{Hb}$ of $\mathrm{Lab} 2$, variation of less than or equal to $2 \mathrm{gm}$ was seen in $73 \%$ patients and more than $2 \mathrm{gm}$ in $24.1 \%$ patients(Table 2).

The mean laboratory $\mathrm{Hb}$ value was $11.9 \pm 2$ for lab 1 and $11.5 \pm 2.1$ for lab 2, mean $\mathrm{SpHb}$ was $12.1 \pm 2.1$ which is greater than lab1 and lab 2(Table 3). The correlation coefficient (r) was 0.588 (Figure 2) and 0.616 for lab1 and 2 against $\mathrm{SpHb}$ and the $\mathrm{p}$ value $<0.001$ represents good correlation between $\mathrm{SpHb}$ and $\mathrm{Hb}$ of Lab1 and $\mathrm{Lab}$ 2(Table 4). The calculated coefficient of determination (r2) was $0.58 \%$. To assess the agreement between the laboratory analyzer and the pulse cooximetry a Bland Altmann plot was applied(Figure 3). The bias and limits of agreement was $0.2 \pm 3.3 \mathrm{gm} / \mathrm{d}$, using this method $95 \%$ of the measurements fell within two standard deviation of the mean difference. The high accuracy(Low ARMSAccuracy root mean square) was obtained for hemoglobin levels less than $12 \mathrm{gm} / \mathrm{dl}$ with an ARMS of $2.52 \mathrm{gm} / \mathrm{dl}$. For hemoglobin between 12-18gm accuracy was low with an ARMS of 4.5 .

Table1: Comparison of SpHb(Pulse co-oximeter) with Hb of Lab1(Sysmex analyzer).

\begin{tabular}{|l|c|}
\hline Hemoglobin & Percentage of patients \\
\hline Difference of $>2$ gms & $20 \%$ \\
\hline Difference of $\leq 2 \mathrm{gms}$ & $75 \%$ \\
\hline Both showed same values & $4.2 \%$ \\
\hline
\end{tabular}

Table 2: Comparison of SpHb(Pulse co-oximeter) with Hb of Lab 2(Medall laboratory analyzer).

\begin{tabular}{|l|c|}
\hline Difference of $>2 \mathrm{gm}$ & $\mathbf{2 4 . 9} \%$ \\
\hline Difference of $\leq 2 \mathrm{gm}$ & $73 \%$ \\
\hline Both showed same values & $2.1 \%$ \\
\hline
\end{tabular}


Table 3: Showing Standard deviation of SpHb, Hb of Lab 1 and Lab2.

\begin{tabular}{|l|c|c|c|}
\hline & Mean & SD & Number \\
\hline $\mathrm{SpHb}$ & 12.1 & 1.39 & 237 \\
\hline $\mathrm{Hb} \mathrm{Lab1}$ & 11.9 & 2.0 & 237 \\
\hline $\mathrm{Hb} \mathrm{Lab2}$ & 11.5 & 2.1 & 237 \\
\hline
\end{tabular}

Table 4: Showing correlative values of SpHb, Lab1, Lab2.

\begin{tabular}{|l|c|c|c|}
\hline \multicolumn{4}{|c|}{ Correlation Values among the Hb of Lab1 and Lab2 with SpHb } \\
\hline Methods & $\mathbf{R}$ & $\mathbf{R 2}$ & P value \\
\hline Lab 1 & 0.589 & 0.347 & $<0.001$ \\
\hline Lab 2 & 0.618 & 0.382 & $<0.001$ \\
\hline
\end{tabular}

Table 5: Showing different studies and their Bias values.

\begin{tabular}{|l|c|c|}
\hline & Number of cases & Bias \\
\hline Weinstein et al ${ }^{12}$ & 710 & 0.03 \\
\hline Hadar et al $^{13}$ & 63 & 0.1 \\
\hline Vora et al $^{14}$ & 76 & 0.2 \\
\hline Macknet et al $^{8}$ & & 0.15 \\
\hline Present study & 237 & 0.2 \\
\hline
\end{tabular}

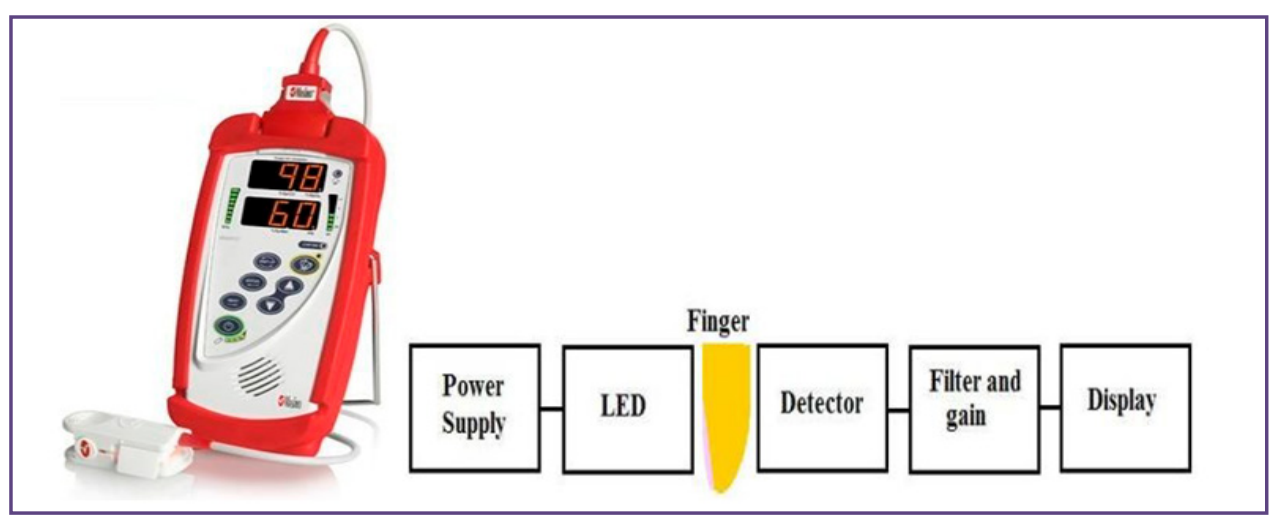

Fig. 1: Pulse Co-oximeter Masimo Rad 57 with displa, figure probe measures SpHb non invasively and basic block disgram ${ }^{4}$.

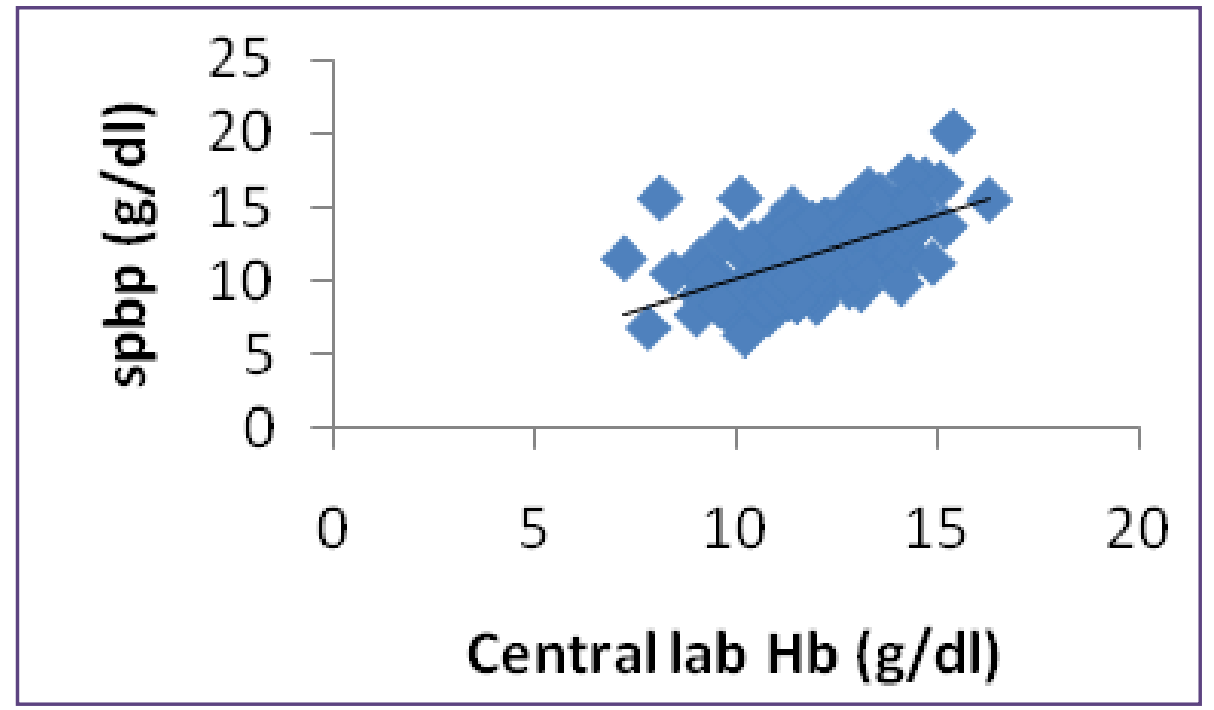

Fig. 2: Scatter plot of hemoglobin values measured by hematology analyzer (lab 1) and pulse co-oximeter. (Correlation coefficient $\mathbf{r}=\mathbf{0 . 5 8 8}$ ). 


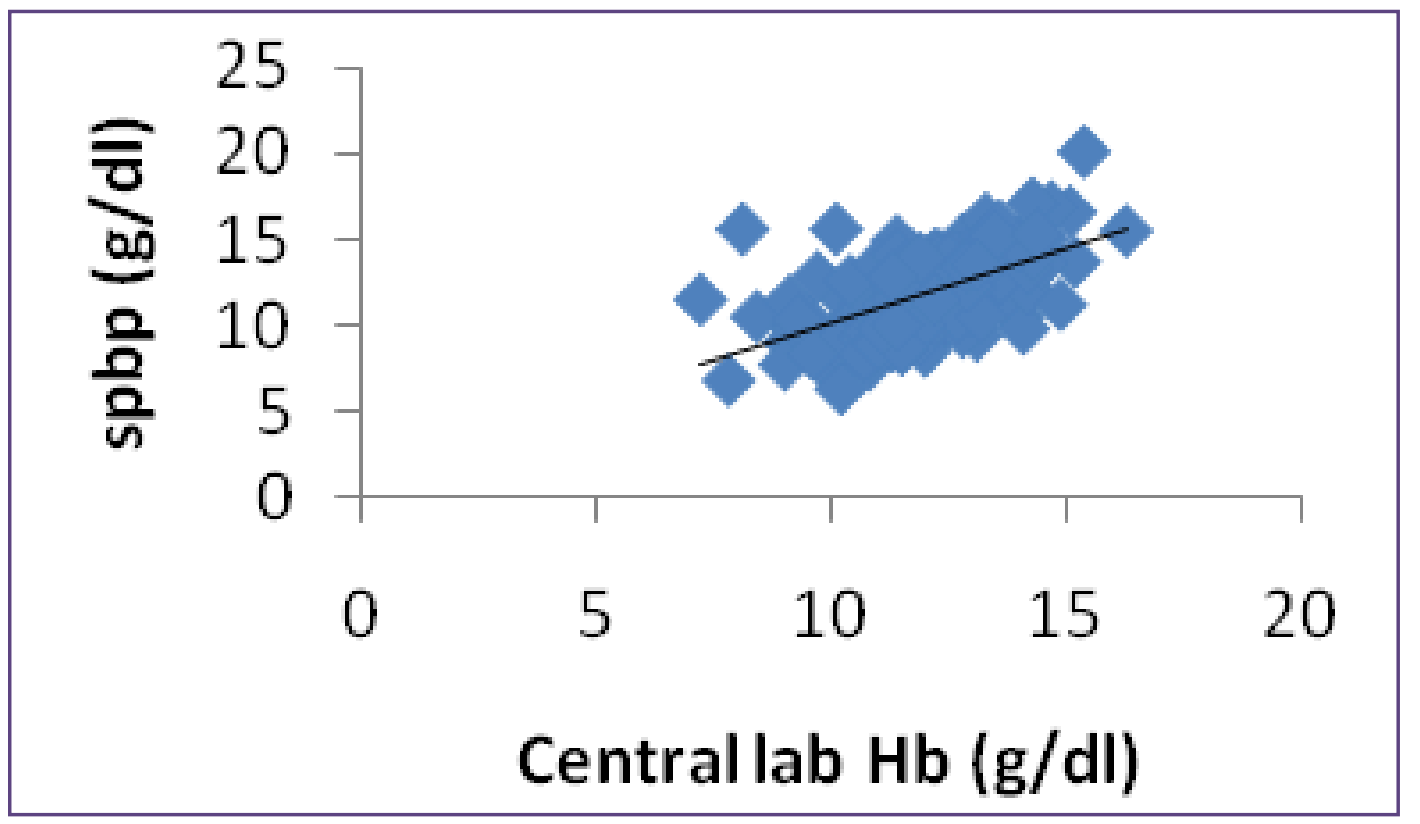

Fig. 3: Bland Altmann representation of comparison analysis between hemoglobin measurement by pulse co-oximeter and hematology analyzer (Lab1). The bias(solid line, 0.2 ) and the limits of agreement (dotted line, bias+1.96SD) are represented on the graph.

\section{Discussion}

The accuracy of pulse co-oximeter device in measuring $\mathrm{Hb}$ was first evaluated in 2007 by Macknet et al ${ }^{[8]}, \mathrm{SpHb}$ provided clinically acceptable accuracy compared with the laboratory $\mathrm{Hb}$ and they were well correlated. In addition there were several reports suggesting that the $\mathrm{SpHb}$ was significantly correlated with the laboratory $\mathrm{Hb}$ during surgical procedures with substantial blood loss ${ }^{[9,10]}$. The present study revealed that noninvasive $\mathrm{SpHb}$ measurement with pulse co-oximetry was significantly correlated with laboratory measurement of $\mathrm{Hb}$.

In 1996, the SET (Signal Extraction Technology) was introduced by Masimo (Irvine, CA) to increase the accuracy of 2 wavelength pulse oximetry under motion and low perfusion conditions ${ }^{[11]}$. Masimo's Rainbow SET technology was later introduced (addition of numerous wavelengths) to measure total $\mathrm{Hb}, \mathrm{COHb}$, and $\mathrm{MetHb}$ concentrations: $\mathrm{CO}-\mathrm{Hb}$ in 2005, MetHb in 2006, and noninvasive $\mathrm{Hb}(\mathrm{SpHb})$ in $2008{ }^{[8]}$. It measures $\mathrm{SpHb}$ using up to 12 wavelengths. Studies have been published evaluating the performance of the Rainbow SET in measuring $\mathrm{SpHb}$ relative to laboratory co-oximeters. The Bland-Altman bias and precision analyses are used to compare 2 technologies. Bias is the mean of the measurement differences between methods, and describes systematic error between measurements (i.e., how closely do results of a new monitor compare to measurements in the laboratory (Table 5). The limits of agreement are defined as the differences between two methods approximately $95 \%$ of the time. The clinically acceptable limits of agreement depend on the variable of interest, the accuracy of the reference standard and what matters clinically.

Nicholas et al ${ }^{[15]}$ studied twenty seven newborn with weight less than 3000 gms. His study showed good correlation between $\mathrm{SpHb}$ and $\mathrm{tHb}(\mathrm{r}=0.75, \mathrm{p}=0.0001)$. The bias and precision for the $\mathrm{Hb}$ and $\mathrm{SpHb}$ values were $0.10 \pm 1.56 \mathrm{~g} / \mathrm{dl}$. A study by Van Woerkom et al ${ }^{[16]}$ using a diffuse optical spectroscopy instrument demonstrated a reliable correlation between tissue haemoglobin and venous haemoglobin before and after a red blood cell transfusion in preterm infants. Torp et al $(\mathrm{n}=471)$ tested the correlation between the Beckman Coulter lab analyzer and the co-oximeter, he found the correlation between the two devices to be $0.93(\mathrm{r})$ with a bias of $0.97 \mathrm{gm} / \mathrm{dl}$.

Beyond the accuracy of $\mathrm{SpHb}$ measurement, another concerning issue is the frequency of events where the $\mathrm{SpHb}$ monitor did not yield data at all or yielded data of low quality. In present study it did not record $\mathrm{SpHb}$ in $9.2 \%$ patients. In his study, Macknet ${ }^{[8]}$ reported the inability to measure $\mathrm{SpHb}$ in $2.4 \%$ of the $\mathrm{SpHb}$ measurements. Gayat et al ${ }^{[17]}$ estimated failure rate to be about 9\% (although the investigators of this study did not adhere to the manufacturer directions or use in the conduct of the 
study). Miller ${ }^{[18]}$ noticed reduced accuracy when the pulse oximeter indicated a low perfusion index. This finding is supported by Gayat et al ${ }^{[17]}$ study where low blood pressure was associated with reduced accuracy. Similar to pulse oximetry, pulse co-oximetry is susceptible to measurement error from the following sources: ambient light interference, low peripheral perfusion, motion artifact, incorrect sensor positioning, nail polish ${ }^{[19]}$. Shielding around the finger probe or photodetector helps to minimize this interference. If there is no detectable peripheral pulsation, the pulse cooximeter cannot function. Hypotension, cold extremities and sever vascular disease are all factors that reduce peripheral pulsations ${ }^{[19]}$. The association between monitor accuracy and peripheral perfusion should not be a surprise, because all pulse oximeters fail to some degree when the patient is peripherally vasoconstricted or hypotensive.

Pulse co-oximeters are good alternate toward reducing iatrogenic blood loss by venipuncture to obtain a blood count. Other draw backs of traditional method are painful needle stick operational inefficiency, delayed $\mathrm{Hb}$ results, potential injury to patient. Additional studies to establish whether the use of this method will potentially reduce iatrogenic blood loss are required. Pulse co-oximeter is a costly instrument and studies are needed to compare both methods on financial perspective also.

Noninvasive pulse co-oximeters are classified as monitoring devices by the FDA(Food and drug administratin) and subsequently do not fall under the $\mathrm{CAP}($ College of American pathologist) and CMS(Centre for medicad services) accreditation of laboratory medicine. Like pulse oximeters, these devices use either a disposable or reusable finger probe. The reusable or "reposable" finger probes are guaranteed for approximately 500 uses. Preventative maintenance is carried out annually using a simulator to verify performance. Routine quality control is not necessary. The device can be operated by a respiratory therapist, registered nurse, certified nursing assistant, or doctor.

\section{Conclusion}

Pulse co-oximeter can be best used as a screening tool for $\mathrm{Hb}$ measurement at out patient departments, blood camps, casuality, ICU and labor rooms. It determines $\mathrm{Hb}$ instantly and non invasively. Further studies are needed to determine financial aspects and needs upgradation in terms of accuracy of the instrument.

\section{Acknowledgements}

I would like to thank all the technical staff of Central laboratory District hospital VIMS Ballari who helped to carry out this study.

\section{Funding}

Hospital funding, Vijayanagara Institute Of Medical Sciences, Ballari Karnataka

\section{Reference}

1. Gayat E, Aulagnier J, Matthieu E, Boisson M, Fischler M. Non-Invasive Measurement of Hemoglobin: Assessment of Two Different Point-of-Care Technologies. PLos ONE . 2012;7(1): 1-8.

2. Zwart A, van Assendelft OW, Bull BS. Recommendations for reference method for haemoglobinometry in human blood (ICSH standard 1995) and specifications for international haemiglobinocyanide standard (4th edition). J Clin Pathol. 1996; 49:271-4.

3. Allard MR, Macknet M, Kherzi S et al: Accuracy of noninvasive hemoglobin measurements by Pulse COOximetry in hemodilution subjects.2009;4:184.

4. Rajashree D, Anagha P. Non-Invasive Optical Sensor for Hemoglobin Determination. IJERA. 2013;3(2):559-62.

5. Bland JM, Altman DG: Agreement between methods of measurement with multipleobservations per individual. J Biopharm Stat. 2007; 17:571-82.

6. Paul BB, Dena MR. Maximizing the laboratory setting for testing devices and understanding statistical output in pulse oximetry. Anesth Analog. 2007; 105:85-94.

7. Frasca D, Dahyot-Fizelier C, Catherine K. Accuracy of a continuous noninvasive hemoglobin monitor in intensive care unit patients. Crit Care Med. 2011; 39:2277-82.

8. Macknet MR, Allard M, Applegate R. The accuracy of noninvasive and continuous total hemoglobin measurement by pulse CO-Oximetry in human subjects undergoing hemodilution. Anesth Analg. 2010; 111:1424-26.

9. Frasca D, Dahyot-Fizelier C, Catherine K. Accuracy of a continuous noninvasive hemoglobin monitor in intensive care unit patients. Crit Care Med. 2011; 39:2277-82.

10. Causey MW, Miller S, Foster A et al: Validation of noninvasive hemoglobin measurements using the Masimo Radical-7 SpHb Station. Am J Sur.j 2011; 201:592-98.

11. Goldman JM, Petterson MT, Kopotic RJ, Barker SJ. Masimo signal extraction pulse oximetry. J Clin Monit Comput. 2000;16:475-83.

12. Ma'ayan L and Choppe L. Non-Invasive Hemoglobin Screening for Diagnosis and Monitoring of Anemia. fnwein@gmail.com.

13. Hadar E, Raban O, Bouganim T, Tenenbaum K, Hod M. Precision and accuracy of noninvasive hemoglobin measurement during pregnancy. The Journal of MaternalFetal and Neonatal Medicine,2012;25(12):2503-06.

14. Vora $H$, Desai $P$. Invasive versus noninvasive hemoglobin measurement by pulse CO-Oximeter in 
neonates admitted to NICU. NHL Journal of Medical Sciences.2014;3(1):68-71.

15. Nicholas C, George R.Validation of non invasive haemoglobin measurement by pulse CO-Oximeter in newborn infants with birth weight less than 3000 gms: pediatric academi societies annual meeting 2013,may 2013.

16. Van Woerkom R, El-Rify ES, Cerussi A, et al. Non-invasive diffuse optical Spectroscopy measures tissue response to red blood cell transfusion in low birth weight infants. Pediatr Res.2003;353:361
17. Gayat E, Bodin A, Sportiello C, Boisson M, Dreyfus JF, Mathieu $\mathrm{E}$ et al. Performance evaluation of a noninvasive hemoglobin monitoring device. Ann Emerg Med. 2011;57:330-3.

18. Miller RD, Ward TA, Shiboski SC, Cohen NH. A comparison of three methods of hemoglobin monitoring in patients undergoing spine surgery. Anesth Analg. 2011;112:858-63.

19. Ruckman JS. A comparative study of total hemoglobin measurement technology: noninvasive pulse cooximetry and conventional methods. Master thesis, University of Connecticut 2011.

*Corresponding author:

Dr. Jayalaxmi Yadav K W/O Somashekar B, \#196 Vishwanathapuram colony, Near govt guest house, Bombay press road, Ballari, Pin code -583101

Karnataka, India

Phone: +919164123763

Email: drjayalaxmiyadav@gmail.com

Date of Submission : 20.06.2017

Date of Acceptance : 11.07.2017

Financial or other Competing Interests: None.

Date of Publication : 30.10.2017 